\title{
A Note on Arbitrage and Closed Convex Cones
}

\author{
Walter Schachermayer* \\ Vienna University of Technology
}

December 3, 2003

\begin{abstract}
We give an example of a subspace $K$ of $L^{\infty}(\Omega, \mathcal{F}, \mathbf{P})$ such that $\bar{K} \cap L_{+}^{\infty}=\{0\}$, where $\bar{K}$ denotes the closure with respect to convergence in probablity. On the other hand, the cone $C:=K-L_{+}^{\infty}$ is dense in $L^{\infty}$ with respect to the weak-star topology $\sigma\left(L^{\infty}, L^{1}\right)$. This example answers a question raised by I. Evstigneev. The topic is motivated by the relation of the notion of no arbitrage and the existence of martingale measures in Mathematical Finance.
\end{abstract}

Key words: Arbitrage, Fundamental Theorem of Asset Pricing.

JEL classification: G10, G12, G13

AMS 2000 subject classifications: Primary 91B24; secondary 91B28, 52A20, $60 \mathrm{H} 30$.

\section{Introduction}

Denote by $(\Omega, \mathcal{F}, \mathbf{P})$ a probability space and recall the following result which plays a basic role in Mathematical Finance.

Theorem 1.1 (Kreps-Yan) Let $1 \leq p \leq \infty$ and $C$ a convex cone in $L^{p}(\Omega, \mathcal{F}, \mathbf{P})$ containing the negative orthant $L_{-}^{p}(\Omega, \mathcal{F}, \mathbf{P})$ and s.t. $C \cap L_{+}^{p}(\Omega, \mathcal{F}, \mathbf{P})=\{0\}$.

If $C$ is closed (w.r. to the norm-topology in the case $1 \leq p<\infty$ and w.r. to the weak-star topology in the case $p=\infty)$ there is an element $g \in L^{q}(\Omega, \mathcal{F}, \mathbf{P})$, where $\frac{1}{p}+\frac{1}{q}=1$, such that $g>0$ a.s. and $\left.g\right|_{C} \leq 0$.

The theorem essentially goes back independently to D. Kreps [K 81] and J.A. Yan [Y 80]. The former author was motivated by Mathematical Finance while the latter was elaborating on the work of Dellacherie and Mokobodzki on the characterisation of semi-martingales. In 1990 C. Stricker ([St 90, Theorem 1 and 2]), extended Yan's result and applied it to Mathematical Finance. For a proof of Theorem 1.1 we refer to [St 90] or [S 94].

The setting in Mathematical Finance to which this theorem applies is roughly as follows: one considers a set $K \subseteq L^{p}(\Omega, \mathcal{F}, \mathbf{P})$ of "contingent claims marketed at price zero". Typically $K$ is defined via

$$
K=\left\{\int_{0}^{T} H_{t} d S_{t} \mid H \in \mathcal{H}\right\}
$$

*Support by the Austrian Science Foundation (FWF) under the Wittgenstein-Preis program Z36MAT and grant P15889, and by the Austrian National Bank under grant 'Jubiläumsfondprojekt Number $9486^{\prime}$ is gratefully acknowledged. I thank an anonymous referee for valuable comments. 
where $S=\left(S_{t}\right)_{0 \leq t \leq T}$ is a semi-martingale modelling the (discounted) price process of one (or several) risky assets and $\mathcal{H}$ ranges through a suitably chosen class of "trading strategies", i.e., predictable $S$-integrable processes. The set $K$ typically is a vector subspace of $L^{p}(\Omega, \mathcal{F}, \mathbf{P})$ or — at least - a convex cone.

The "principle of no arbitrage" is the following assumption on $K$ :

$$
\text { (NA) } K \cap L_{+}^{p}(\Omega, \mathcal{F}, \mathbf{P})=\{0\} .
$$

The interpretation is that it should not be possible to find a contingent claim marketed at price zero, i.e., an element $f \in K$, such that $f \geq 0$ a.s. and $\mathbf{P}[f>0]>0$, as this would yield an arbitrage opportunity.

The Fundamental Theorem of Asset Pricing states that the condition (NA) is essentially equivalent to the existence of a linear functional $g \in L^{q}(\Omega, \mathcal{F}, \mathbf{P}), g>0$ a.s. such that $\left.g\right|_{K} \leq 0$ which, in the case when $K$ is a vector space, is equivalent to $\left.g\right|_{K}=0$.

In the case of finite $\Omega$ and defining $K$ as in (1), where the class $\mathcal{H}$ consists of all predictable processes, this is indeed a mathematical theorem (i.e., the word "essentially" above may simply be dropped), which is due to Harrison and Pliska [HP 81]. The (properly normalized) linear functional $g$ may then be interpreted as the density $\frac{d \mathbf{Q}}{d \mathbf{P}}$ of a probability measure $\mathbf{Q}$ equivalent to $\mathbf{P}$ under which the process $S$ is a martingale.

Turning to more general situations than finite probability spaces $\Omega$, it was noticed by $\mathrm{D}$. Kreps [K 81] that a strengthening of the $(N A)$ condition is needed in order to give the word "essentially" above a precise meaning. The idea is to replace the condition $K \cap L_{+}^{p}=\{0\}$ by $\widetilde{K} \cap L_{+}^{p}=\{0\}$, where $\widetilde{K}$ is an enlargement of $K$ in an appropriate sense. At this stage D. Kreps observed a remarkable phenomenon: to obtain $\widetilde{K}$ it is the wrong idea to try to pass directly to a topological closure of $K$. The good idea is first to pass to the cone

$$
\begin{aligned}
C & =K-L_{+}^{p}(\Omega, \mathcal{F}, \mathbf{P}) \\
& =\left\{f \in L^{p}(\Omega, \mathcal{F}, \mathbf{P}) \mid \text { there is } h \in K \text { with } f \leq h\right\} .
\end{aligned}
$$

Note that the no arbitrage condition $K \cap L_{+}^{p}=\{0\}$ is obviously equivalent to $C \cap L_{+}^{p}=$ $\{0\}$. The economic interpretation of the passage from $K$ to $C$ is that "agents are allowed to throw away money", or the hypothesis of "free disposal".

Somewhat surprisingly, this - apparently silly — passage from $K$ to $C$ changes the situation dramatically when we now pass to taking topological closures.

Denoting by $\bar{C}$ the closure of $C$ in an appropriate topology (in the present setting the norm topology of $L^{p}$ for $1 \leq p<\infty$ and the weak-star topology for $p=\infty$ ), D. Kreps [K 81] introduces the condition of "no free lunch"

$$
(N F L) \quad \bar{C} \cap L_{+}^{p}(\Omega, \mathcal{F}, \mathbf{P})=\{0\} .
$$

and gives an economic interpretation as a strengthening of the no arbitrage condition $(N A)$. Now he is in a position to apply Theorem 1.1 to obtain a linear functional $g>0$ such that $\left.g\right|_{C} \leq 0$, which enables him to state and prove a mathematically precise version of the Fundamental Theorem of Asset Pricing. Many authors have elaborated further on this seminal work by D. Kreps (see, e.g., [DS 94], [DS 98] and the references given there).

But what goes wrong if one tries to replace the condition (NFL) by

$$
\bar{K} \cap L_{+}^{p}(\Omega, \mathcal{F}, \mathbf{P})=\{0\},
$$

which - intuitively speaking - may seem to yield the same condition as (NFL) (the bars pertaining to the same topology)? In Example 3.1 of [S 94] a subspace $K$ of 
$L^{\infty}(\Omega, \mathcal{F}, \mathbf{P})$ was constructed along the lines of $(1)$ such that, letting $C=K-L_{+}^{\infty}$ and denoting by $\bar{K}$ and $\bar{C}$ the respective closures in the weak-star topology of $L^{\infty}$, equality (5) holds true while $\bar{C}$ equals the entire space $L^{\infty}$ (so that, in particular, (4) fails to hold true). In fact the example is such that $\bar{K}^{\|\cdot\|_{1}} \cap L_{+}^{1}(\Omega, \mathcal{F}, \mathbf{P})=\{0\}$ holds true, where $\bar{K}^{\|\cdot\|_{1}}$ denotes the closure of $K$ in the norm topology of $L^{1}(\Omega, \mathcal{F}, \mathbf{P})$. This is a stronger assertion than $\bar{K} \cap L_{+}^{\infty}(\Omega, \mathcal{F}, \mathbf{P})=\{0\}$.

The purpose of this note is to investigate the above described phenomenon a little further and to push it to the maximal limits in the following sense.

Theorem 1.2 There is a vector space $K$ in $L^{\infty}(\Omega, \mathcal{F}, \mathbf{P})$ where $\Omega=[0,1], \mathcal{F}=$ Borel $[0,1]$, and $\mathbf{P}$ equals Lebesgue measure $\lambda$ on $\mathcal{F}$, such that, letting $C=K-L_{+}^{\infty}$, the following statements hold true:

(i) $\bar{K} \cap L_{+}^{0}(\Omega, \mathcal{F}, \mathbf{P})=\{0\}$, where $\bar{K}$ denotes the closure of $K$ with respect to the topology of convergence in probability.

(ii) $\bar{C} \cap L_{+}^{\infty}(\Omega, \mathcal{F}, \mathbf{P}) \neq\{0\}$, where $\bar{C}$ denotes the closure of $C$ with respect to the norm topology of $L^{\infty}$.

More precisely, for $\varepsilon>0$, there is $f \in \bar{C}$, taking its values a.s. in $\{0,1\}$ and s.t. $\mathbf{P}[f=1]>1-\varepsilon$. In particular, $C$ is weak-star dense in $L^{\infty}$.

For the definition of $\bar{K}$ we have considered the coarsest conceivable topology, while for $\bar{C}$ the finest conceivable one in the present context. We still can observe the above described phenomenon that (4) fails while (5) holds true.

One might ask whether one may push in (ii) still a little further to obtain an example where $C$ is dense in $L^{\infty}$ with respect to the norm $\|\cdot\|_{\infty}$. However, a moment's reflexion reveals that this is asking for too much: if the constant function $\mathbf{1}$ is in the closure of $C$ with respect to $\|\cdot\|_{\infty}$, we cannot have $K \cap L_{+}^{\infty}=\{0\}$. Hence it seems that the above theorem precisely shows how far the above described phenomenon can be pushed.

I. Evstigneev has informed me [E 02] that he has constructed a convex cone $K$ in $L^{1}$ displaying similar phenomena as the space $K$ described in Theorem 1.2 above. He asked whether the convex cone can be replaced by a vector space.

It turns out that, using wellknown properties of $\alpha$-stable random variables the construction of such a space is relatively simple. A similar idea was already used in the thesis of W. Brannath [B 97] in the context of "immediate free lunches"; there, however, a sequence of "taylor-made" random variables was used instead of the $\alpha$ stable ones we shall presently use.

In the subsequent section we give the proof of Theorem 1.2.

\section{The Example}

Using the notation of ([ST 94, Definition 1.1.6.]), fix $0<\alpha<1, \sigma>0$ and let $X$ be an $\alpha$-stable random variable with scale parameter $\sigma$, skewness parameter $\beta=1$ and shift parameter $\mu=0$, i.e. $X \sim S_{\alpha}(\sigma, 1,0)$. Let $\left(X_{n}\right)_{n=1}^{\infty}$ be a sequence of i.i.d. copies of $X$. The random variables $\left(X_{n}\right)_{n=1}^{\infty}$ are defined on a stochastic base $(\Omega, \mathcal{F}, \mathbf{P})$ which we may assume w.l.g. to equal $([0,1]$, Borel $[0,1], \lambda)$.

We need the following properties of $X$ (see sections 1.1 and 1.2 of [ST 94]):

(i) The support of $X$ equals $\mathbb{R}_{+}$. 
(ii) For $N \in \mathbb{N}$ and $\xi=\left(\xi_{1}, \ldots, \xi_{N}\right) \in \mathbb{R}_{+}^{N}$, the r.v. $\sum_{n=1}^{N} \xi_{n} X_{n}$ has the same distribution as $\|\xi\|_{\alpha} X$, where $\|\xi\|_{\alpha}=\left(\sum_{n=1}^{N}\left|\xi_{n}\right|^{\alpha}\right)^{\frac{1}{\alpha}}$.

Denoting by $\left(\ell^{\alpha},\|\cdot\|_{\alpha}\right)$ the quasi-normed complete metric vector space of all sequences $\xi=\left(\xi_{n}\right)_{n=1}^{\infty}$ such that $\|\xi\|_{\alpha}=\left(\sum_{n=1}^{\infty}\left|\xi_{n}\right|^{\alpha}\right)^{\frac{1}{\alpha}}<\infty$, property (ii) implies the following well-known result.

Lemma 2.1 The mapping

$$
\begin{aligned}
i: \quad \ell^{\alpha} & \rightarrow L^{0}(\Omega, \mathcal{F}, \mathbf{P}) \\
\left(\xi_{n}\right)_{n=1}^{\infty} & \mapsto \sum_{n=1}^{\infty} \xi_{n} X_{n}
\end{aligned}
$$

is a well-defined isomorphic embedding of the quasi-normed topological vector space $\left(\ell^{\alpha},\|\cdot\|_{\alpha}\right)$ into the topological vector space $L^{0}(\Omega, \mathcal{F}, \mathbf{P})$, equipped with the topology of convergence in probability.

Proof. Write $\xi=\xi^{+}-\xi^{-}$, where $\xi^{+}=\left(\xi_{n}^{+}\right)_{n=1}^{\infty}$ and $\xi^{-}=\left(\xi_{n}^{-}\right)_{n=1}^{\infty}$ and note that $\|\xi\|_{\alpha} \geq \max \left(\left\|\xi^{+}\right\|_{\alpha},\left\|\xi^{-}\right\|_{\alpha}\right) \geq 2^{-1 / \alpha}\|\xi\|_{\alpha}$.

We infer from property (ii) above that $i\left(\xi^{+}\right)$(resp. $i\left(\xi^{-}\right)$) have the same distribution as $\left\|\xi^{+}\right\|_{\alpha} X$ (resp. $\left.\left\|\xi^{-}\right\|_{\alpha} X\right)$. As these two random variables are independent the assertion of the lemma follows.

We shall apply Lemma 2.1 in a slightly modified form: Let $\left(c_{n}\right)_{n=1}^{\infty}$ be a sequence of real numbers and define

$$
Y_{n}=X_{n}-c_{n}
$$

Lemma 2.2 If the sequence $\left(c_{n}\right)_{n=1}^{\infty}$ is bounded, the mapping

$$
\begin{aligned}
j: \quad \ell^{\alpha} & \rightarrow L^{0}(\Omega, \mathcal{F}, \mathbf{P}) \\
\left(\xi_{n}\right)_{n=1}^{\infty} & \mapsto \sum_{n=1}^{\infty} \xi_{n} Y_{n}
\end{aligned}
$$

shares all the properties of the map $i$ indicated in Lemma 2.1

Proof of Lemma 2.2. To show the continuity of $j$ note that

$$
\begin{aligned}
\varphi: \quad \ell^{\alpha} & \rightarrow \mathbb{R} \\
\left(\xi_{n}\right)_{n=1}^{\infty} & \mapsto \sum_{n=1}^{\infty} c_{n} \xi_{n}
\end{aligned}
$$

is a continuous linear functional on $\left(l^{\alpha},\|\cdot\|_{\alpha}\right)$ if $\left(c_{n}\right)_{n=1}^{\infty}$ is a bounded sequence of real numbers. As $j=i+\psi \varphi$, where $\psi: \mathbb{R} \rightarrow L^{0}(\Omega, \mathcal{F}, \mathbf{P})$ is the linear embedding defined by $\psi(1)=-\mathbf{1}$, we obtain the continuity of $j$ from the continuity of $i$.

To show the openess of the mapping $j$ from $\ell^{\alpha}$ onto its image in $L^{0}(\Omega, \mathcal{F}, \mathbf{P})$ we have to show that for $\varepsilon>0$ we may find $\delta>0$ such that $\|\xi\|_{\alpha}>\varepsilon$ implies that $\mathbf{P}[j(\xi)>\delta]>\delta$. Writing again $\xi=\xi^{+}-\xi^{-}$, we have

$$
j(\xi) \sim\left\|\xi^{+}\right\|_{\alpha} X_{1}-\left\|\xi^{-}\right\|_{\alpha} X_{2}-\sum_{n=1}^{\infty} c_{n} \xi_{n}
$$

where $\sim$ denotes equality in distribution, and we may conclude the assertion similarly as in Lemma 2.1 above. 
From now on we shall specify the choice of $c_{n}$ by $c_{n}=n^{-1}$. Denoting by $K_{0}$ the image $j\left(l^{\alpha}\right)$ in $L^{0}(\Omega, \mathcal{F}, \mathbf{P})$, we shall see that this vector space has already essentially the properties listed in Theorem 1.2 (with the exception that it lives in $L^{0}$ instead of $\left.L^{\infty}\right)$.

Indeed, $K_{0}$ is a closed subspace of $L^{0}(\Omega, \mathcal{F}, \mathbf{P})$ by Lemma 2.2. Next we show that

$$
K_{0} \cap L_{+}^{0}(\Omega, \mathcal{F}, \mathbf{P})=\{0\} .
$$

Fix $\xi=\left(\xi_{n}\right)_{n=1}^{\infty} \in l^{\alpha}, \xi \neq 0$, and write again $\xi=\xi^{+}-\xi^{-}$.

If $\xi^{+} \neq 0$ then by property (i) above the support of the random variable $j\left(\xi^{+}\right)$ equals $\left[-\sum_{n=1}^{\infty} \frac{\xi_{n}^{+}}{n}, \infty[\right.$.

Similarly, if $\xi^{-} \neq 0$, the support of $j\left(-\xi^{-}\right)$contains a non-empty subset of $]-\infty, 0[$ (in fact it contains ] $-\infty, 0]$, but for later use we note that it is sufficient for the subsequent argument that it contains a non-empty subset of $]-\infty, 0[)$. As $j(\xi)$ is the sum of the two independent r.v. $j\left(\xi^{+}\right)$and $j\left(-\xi^{-}\right)$we conclude that $\xi \neq 0$ implies that the intersection of the support of $j(\xi)$ with ] - $-0,0$ [is not empty, thus proving (14).

As regards assertion (ii) of Theorem 1.2 we have the following result.

Lemma 2.3 Denote by $C_{0}$ the cone $\left(K_{0}-L_{+}^{0}(\Omega, \mathcal{F}, \mathbf{P})\right) \cap L^{\infty}(\Omega, \mathcal{F}, \mathbf{P})$ and by $\bar{C}_{0}$ its closure w.r. to $\|.\|_{\infty}$. For $\varepsilon>0$ there is $f \in \bar{C}_{0}$ taking its values in $\{0,1\}$ such that $\mathbf{P}[f=1]>1-\varepsilon$.

Proof of Lemma 2.3. We first show that, for $\delta>0$, there is $g \in C_{0}$ taking its values in $[-\delta, 1]$ such that $\mathbf{P}[g=1]>1-\delta$. Fix $\delta>0$, let $M>0$ be big enough such that $\mathbf{E}[X \wedge M]>1$ and $N_{\delta} \in \mathbb{N}$ such that $N_{\delta}^{-1}<\delta$. For $N>N_{\delta}$ let

$$
f_{N, \delta}=\frac{1}{N-N_{\delta}} \sum_{n=N_{\delta}+1}^{N}\left(Y_{n} \wedge M\right) .
$$

Then $f_{N, \delta}$ is in $C_{0}$, takes its values in $[-\delta, M]$ and, by the strong law of large numbers, tends for $N \rightarrow \infty$ almost surely to $\mathbf{E}[X \wedge M]$. Hence, taking $N$ sufficiently large and letting $g=f_{N, \delta} \wedge 1$ we have found a function $g \in C_{0}$ as indicated above.

To show the assertion of the Lemma fix $\varepsilon>0$ and find a sequence $\left(g_{n}\right)_{n=1}^{\infty} \in C_{0}$ as above with $\delta=\frac{\varepsilon}{2^{n}}$. Letting $A_{n}=\left\{g_{n}=1\right\}$ and $A=\bigcap_{n=1}^{\infty} A_{n}$ we find $\mathbf{P}[A]>1-\varepsilon$. We have that $f=\mathbf{1}_{A}$ is in $\bar{C}_{0}$ as $g_{n} \wedge \mathbf{1}_{A} \in C_{0}$ and $\left\|f-\left(g_{n} \wedge \mathbf{1}_{A}\right)\right\|_{\infty} \leq \frac{\varepsilon}{2^{n}}$.

The only step we are still missing in order to show the assertions of Theorem 1.2 is to replace the subspace $K_{0}$ of $L^{0}(\Omega, \mathcal{F}, \mathbf{P})$ by an analogous subspace $K$ of $L^{\infty}(\Omega, \mathcal{F}, \mathbf{P})$. This will be done by a straightforward truncation argument.

Let $\left(M_{n}\right)_{n=1}^{\infty}$ be a sequence of strictly positive numbers tending sufficiently fast to infinity so that

$$
\sum_{n=1}^{\infty} \mathbf{P}\left[X>M_{n}\right]<\infty .
$$

Let $Z_{n}=Y_{n} \wedge M_{n}$ and define $K$ as the linear span of the sequence $\left(Z_{n}\right)_{n=1}^{\infty}$ in $L^{\infty}(\Omega, \mathcal{F}, \mathbf{P})$. We claim that $K$ satisfies all the requirements of Theorem 1.2.

Lemma 2.4 Denoting by $\bar{K}$ the closure of $K$ in $L^{0}(\Omega, \mathcal{F}, \mathbf{P})$ with respect to the topology of convergence in measure and letting

$$
\begin{aligned}
k: \quad \ell^{\alpha} & \rightarrow L^{0}(\Omega, \mathcal{F}, \mathbf{P}) \\
\left(\xi_{n}\right)_{n=1}^{\infty} & \mapsto \sum_{n=1}^{\infty} \xi_{n} Z_{n},
\end{aligned}
$$


we have that $k$ is a well-defined isomorphic embedding. In particular, we have $k\left(l^{\alpha}\right)=$ $\bar{K}$.

Proof. To show the continuity of $k$ denote by $k_{N}$ the map $k_{N}: l^{\alpha} \rightarrow L^{0}$ given by $k_{N}(\xi)=\sum_{n=1}^{N} \xi_{n} Z_{n}$. We infer from Lemma 2.2, (16) and the Borel-Cantelli Lemma that, for each $\xi \in l^{\alpha}$, the sequence $\left(k_{N}(\xi)\right)_{N=1}^{\infty}$ converges in probability. This shows that $k$ is well-defined and we infer from the uniform boundedness principle (applied to sequences of operators from the complete metric topological vector space $\left(l^{\alpha}\|\cdot\|_{\alpha}\right)$ to the topological vector space $\left.L^{0}(\Omega, \mathcal{F}, \mathbf{P})\right)$, that $k$ is continuous.

To show that $k$ is an isomorphic embedding let $\left(\xi^{i}\right)_{i=1}^{\infty}=\left(\left(\xi_{n}^{i}\right)_{i=1}^{\infty}\right)_{n=1}^{\infty}$ be a sequence in $l^{\alpha}$ such that $\left\|\xi^{i}\right\|_{\alpha}=1$. Assuming that $k\left(\xi^{i}\right)$ tends to zero in $L^{0}(\Omega, \mathcal{F}, \mathbf{P})$ we have to arrive at a contradiction.

We distinguish two cases. Either there is some $n_{0}$ such that $\left(\xi_{n_{0}}^{i}\right)_{i=1}^{\infty}$ does not tend to zero. In this case we may suppose, by passing to a subsequence, that $\left(\xi_{n_{0}}^{i}\right)_{i=1}^{\infty}$ converges to some $\xi_{n_{0}} \neq 0$. The random variable $k\left(\xi^{i}\right)$ then is the sum of the two independent random variables $\xi_{n_{0}}^{i} Z_{n_{0}}$ and $\sum_{n \neq n_{0}} \xi_{n}^{i} Z_{n}$. As the former sequence $\left(\xi_{n_{0}}^{i} Z_{n_{0}}\right)_{i=1}^{\infty}$ converges to the non-degenerate random variable $\xi_{n_{0}} Z_{n_{0}}$ we readily see that $\left(k\left(\xi^{i}\right)\right)_{i=1}^{\infty}$ cannot tend to zero in probability.

The second case is when $\left(\xi_{n}^{i}\right)_{i=1}^{\infty}$ tends to zero, for each fixed $n \in \mathbb{N}$. In this case we infer from (16) and the Borel-Cantelli Lemma that $\left(k\left(\xi^{i}\right)-j\left(\xi^{i}\right)\right)_{i=1}^{\infty}$ converges to zero a.s. and therefore in probability. Hence we may conclude as in (13) above that $\left(k\left(\xi^{i}\right)\right)_{i=1}^{\infty}$ cannot converge to zero in probability.

Lemma 2.5 The subspace $K$ of $L^{\infty}(\Omega, \mathcal{F}, \mathbf{P})$ defined after (16) satisfies the assertions of Theorem 1.2.

Proof. As regards assertion (i) of Theorem 1.2 we have seen in Lemma 2.4 that $k\left(\ell^{\alpha}\right)=\bar{K}$. To verify that $\bar{K} \cap L_{+}^{0}(\Omega, \mathcal{F}, \mathbf{P})=\{0\}$ it therefore suffices to repeat the argument after (14).

As regards the existence of $f \in \bar{C}$ with the properties indicated in Theorem 1.2, one may repeat verbatim the proof of Lemma 2.3 (by making sure that $N_{\delta} \in \mathbb{N}$ is large enough so that $M_{n} \geq M$ for $n \geq N_{\delta}$ ).

The weak-star closure of $C$ therefore contains the constant function $\mathbf{1}$, is a cone and contains $L_{-}^{\infty}(\Omega, \mathcal{F}, \mathbf{P})$, hence it must be equal to the entire space $L^{\infty}(\Omega, \mathcal{F}, \mathbf{P})$.

Summing up, we have proved all the assertions of Theorem 1.2

Finally, let us interpret the above constructed vector space $K$ as a space of stochastic integrals. This is an easy task by simply translating the above construction into a one period financial market with countably many assets. More formally, let $S=\left(S_{t}\right)_{t=0}^{1}$ be the $\mathbb{R}^{\mathbb{N}}$-valued process defined by $S_{0}=0$ and $S_{1}=\left(Z_{n}\right)_{n=0}^{\infty}$.

This process is based on $(\Omega, \mathcal{F}, \mathbf{P})=([0,1]$, Borel $[0,1], \lambda)$ and the filtration $\left(\mathcal{F}_{t}\right)_{t=0}^{1}$ is given by $\mathcal{F}_{0}=\{\emptyset, \Omega\}$ and $\mathcal{F}_{1}=\mathcal{F}$.

Putting $\mathbb{R}^{\mathbb{N}}$ in duality with the space $\mathbb{R}^{(\mathbb{N})}$ of finite sequences, we obtain as the space of stochastic integrals (1) precisely the linear combinations of $\left(Z_{n}\right)_{n=1}^{\infty}$, i.e., the space $K$ defined after (16) above. The financial market $S$ therefore does not permit an equivalent martingale measure as it allows for free lunches; more precisely, and using the terminology from [DS 94], it allows for a free lunch with vanishing risk by Theorem 1.2 (ii). On the other hand, it is not possible to approximate a non-negative claim $f \in L_{+}^{\infty}(\Omega, \mathcal{F}, \mathbf{P}) \backslash\{0\}$ by elements in $K$, not even in probability (Theorem $1.2(\mathrm{i}))$. We thus encounter a problem of "embarras de richesse", if agents are not allowed to "throw away money".

We still remark that it is not as obvious how to transform the above construction into an example of an $\mathbb{R}$-valued (or $\mathbb{R}^{d}$-valued, for some $1 \leq d<\infty$ ) process $S$ such 
that the space of stochastic integrals displays similar phenomena as the space $K$ above. We leave this question for further research.

\section{References}

[B 97] W. Brannath, (1997), On fundamental theorems in mathematical finance. Doctoral Thesis, University of Vienna.

[DS 94] F. Delbaen, W. Schachermayer, (1994), A General Version of the Fundamental Theorem of Asset Pricing. Math. Annalen, Vol. 300, pp. 463-520.

[DS 98] F. Delbaen, W. Schachermayer, (1998), The Fundamental Theorem of Asset Pricing for Unbounded Stochastic Processes. Mathematische Annalen, Vol. 312, pp. 215-250.

[E 02] I. Evstigneev, (2002), Personal communication.

[HP 81] J.M Harrison, S.R. Pliska, (1981), Martingales and Stochastic integrals in the theory of continuous trading. Stoch. Proc. \& Appl., Vol. 11, pp. 215-260.

[K 81] D.M. Kreps, (1981), Arbitrage and equilibrium in economies with infinitely many commodities. J. Math. Econ., Vol. 8, pp. 15-35.

[S 94] W. Schachermayer, (1994), Martingale Measures for discrete time processes with infinite horizon. Math. Finance, Vol. 4, No. 1, pp. 25-55.

[St 90] C. Stricker, (1990), Arbitrage et lois de martingale. Ann. Inst. H.Poincaré Probab. Statist., Vol. 26, pp. 451-460.

[ST 94] G. Samorodnitsky, M.S.Taqqu, (1994), Stable Non-Gaussian Random Processes. Stochastic Models with Infinite Variance Chapman\& Hall.

[Y 80] J.A. Yan, (1980), Caracterisation d' une classe d'ensembles convexes de $L^{1}$ ou $H^{1}$. Lect. Notes Mathematics, Vol. 784, pp. 220-222. 